\title{
OBESIDADE: ATUALIZAÇÃO SOBRE SUA ETIOLOGIA, MORBIDADE E TRATAMENTO
}

\section{OBESITY: UPDATED INFORMATION ABOUT ITS ETIOLOGY, MORBIDITY AND TREAT- MENT}

\author{
Rachel Pamfilio Prado de FRANCISCHI ${ }^{1,2}$ \\ Luciana Oquendo PEREIRA ${ }^{3}$ \\ Camila Sanchez FREITAS 2 \\ Mariana KLOPFER ${ }^{2}$ \\ Rogério Camargo SANTOS 4 \\ Patrícia VIEIRA ${ }^{4}$ \\ Antônio Herbert LANCHA JÚNIOR 5
}

\section{RESUMO}

\begin{abstract}
As tendências de transição nutricional ocorridas neste século direcionam para uma dieta mais ocidentalizada, a qual, aliada à diminuição progressiva da atividade física, converge para o aumento no número de casos de obesidade em todo o mundo. Isso representa aumento na morbidade e na mortalidade associadas à obesidade, já que esta é fator de risco para várias doenças como diabetes tipo II, hipertensão, doenças cardiovasculares e cálculo na vesícula biliar. A obesidade se apresenta não apenas como problema científico e de saúde pública, porém como grande indústria que envolve o desenvolvimento de fármacos, de alimentos modificados e estratégias governamentais estimulando a prática regular de atividade física e a orientação alimentar a fim de promover melhores hábitos. Assim, o conhecimento das causas e estratégias preventivas da obesidade é o objeto de estudo de pesquisadores de diferentes centros. Esse artigo tem como objetivo rever esses estudos, abordando o aumento na prevalência e incidência da obesidade, doenças relacionadas ao excesso de peso e os tratamentos para redução da gordura corporal.
\end{abstract}

Termos de indexação: obesidade, etiologia, dietoterapia, diabetes mellitus não insulino-dependente, morbidade.

\section{ABSTRACT}

The trend in nutritional transition in this century leads to an occidentalized diet, which, allied to a decrease in physical activity, results in increasing of obesity all over the world. It raises the

\footnotetext{
(1) Bolsista de Iniciação Científica da Fundação de Amparoà Pesquisa do Estado de São Paulo (FAPESP) do Laboratório de Nutrição e Metabolismo Aplicadoà Atividade Motora, Escola de Educação Física e Esporte, Universidade de São Paulo.

(2) Curso de Nutrição, Faculdade de Saúde Pública, Universidade de São Paulo. Av. Prof. Mello de Moraes, 65, 05508-900. São Paulo, SP, Brasil. Correspondência para/ Correspondenceto: R.P.P.FRANCISCHI.E-mail: racnutri@usp.br

(3) Departamento de Bioquímica, Instituto de Biologia, Universidade Estadual de Campinas.

(4) Laboratório de Nutrição e Metabolismo Aplicadoà Atividade Motora, Escola de Educação Física e Esporte, Universidade de São Paulo.

(5) Escola de Educação Física e Esporte, Universidade deSão Paulo.
} 
risk of morbidity and mortality, since obesity is the first step to several diseases such as Diabetes type II, hypertension, cardiovascular and gallbladder diseases. Obesity is not only a scientific and public health problem, but also an industry of pharmacos, special foods and governmental strategies to encourage people to be more active and to provide more food information in order

to promote better habits. Several studies all around the world discuss the causes and treatments for obesity. In this way, this paper summarizes these researches, approaching the elements associated with higher obesity incidence and prevalence, the diseases related to overweight and the treatments for reducing body fat.

Index terms: obesity, etiology, diet therapy, diabetes millitus, noninsulin-dependente, morbidity.

\section{INTRODUÇÃO}

A obesidade é provavelmente o mais antigo distúrbio metabólico, havendo relatos da ocorrência desta desordem em múmias egípcias e em esculturas gregas (Blumenkrantz, 1997). Recentemente, a obesidade pôde ser considerada a mais importante desordem nutricional nos países desenvolvidos, tendo em vista o aumento de sua incidência: acredita-se que atinja $10 \%$ da população desses países (Dyer, 1994) e que mais de um terço da população norte-americana esteja acima do peso desejável (Baron, 1995). A obesidade está sendo considerada uma epidemia mundial, presente tanto em países desenvolvidos como em desenvolvimento (Popkin \& Doak, 1998). O aumento de sua incidência está distribuído em quase todas as raças e sexos, e atinge principalmente a população de 25 a 44 anos (Blumenkrantz, 1997).

Pode-se afirmar que as tendências de transição nutricional ocorrida neste século em diferentes países do mundo convergem para uma dieta mais rica em gorduras (particularmente as de origem animal), açúcares e alimentos refinados, e reduzida em carboidratos complexos e fibras, também conhecida como "dieta ocidental" (Monteiro et al., 1995). Aliando esse fator com o declínio progressivo da atividade física dos indivíduos, percebe-se alterações concomitantes na composição corporal, principalmente o aumento da gordura. No caso do Brasil, estudos comprovam que essa transição nos padrões nutricionais, relacionando-os com mudanças demográficas, socioeconômicas e epidemiológicas ao longo do tempo, estão refletindo na diminuição progressiva da desnutrição e no aumento da obesidade (Monteiro et al., 1995). Dados divulgados pela Sociedade Brasileira de Cardiologia apontam que $80 \%$ da nossa população adulta é sedentária e que $32 \%$ dos adultos brasileiros são obesos (Sociedade..., 1999a,b).

Monteiro et al. (1995) encontraram informações importantes quanto ao aumento da prevalência da obesidade no Brasil, principalmente quando se considera que este aumento, apesar de estar distribuído em todos os estratos econômicos da população, é proporcionalmente mais elevado nas famílias de mais baixa renda. Isso também pode ser observado nos Estados Unidos, já que essa desordem nutricional atinge a sua população independente do nível socioeconômico, sendo predominantemente encontrada entre as pessoas de classe econômica baixa (Bray, 1992).

Ao estudar a obesidade e sua evolução, é certo admitir que o seu aumento implica definição de prioridades e estratégias de ação de Saúde Pública, em especial à prevenção e ao controle das doenças crônicas, reservando lugar de destaque à ações de educação em alimentação e nutrição e as práticas de atividades físicas que alcancem de forma eficaz todas as camadas sociais da população (Monteiro et al., 1995).

Desse modo, este artigo tem como objetivo descrever os aspectos gerais da obesidade, abordando de forma sucinta e atualizada os elementos chaves ligados ao aumento na sua prevalência e incidência, doenças relacionadas ao excesso de peso e os tratamentos mais utilizados para a redução da gordura corporal.

\section{CAUSAS DA OBESIDADE}

A obesidade não é uma desordem singular, e sim um grupo heterogêneo de condições com múltiplas causas que em última análise resultam no fenótipo de obesidade. Os princípios mendelianos e a influência do genótipo na etiologia desta desordem podem ser atenuados ou exacerbados por fatores não-genéticos, como o ambiente externo e interações psicossociais que atuam sobre mediadores fisiológicos de gasto e consumo energético (Jebb, 1997). Segundo a Organização Mundial da Saúde (OMS) (World..., 1990), a ocorrência da obesidade nos indivíduos reflete a interação entre fatores dietéticos e ambientais com uma predisposição genética. Contudo, existem poucas evidências de que algumas populações são mais suscetíveis à obesidade por motivos genéticos, o que reforça serem os fatores alimentares - em especial a dieta e a atividade física - responsáveis pela diferença na prevalência da obesidade em diferentes grupos populacionais (World... 1990).

Dentre os fatores alimentares, pode-se destacar o excesso de energia e, principalmente, de lipídeos, favorecendo o aumento da adiposidade (World..., 1990; 
Rolls \& Shide, 1992). Recentes estudos com mulheres obesas brasileiras têm apontado a alta ingestão de lipídeos, muito freqüente nessa população, apesar da amostra considerada ser pequena para predizer níveis populacionais (Pereira, 1998; Francischi et al., 1999). Outro aspecto alimentar ressaltado por Jebb (1997) é quanto a freqüência alimentar, já que os indivíduos que consomem maior número de pequenas refeições ao longo do dia apresentam peso relativamente menor do que aqueles que consomem número menor de grandes refeições. Quanto à prática de exercícios físicos, já é consenso que a medida que a sociedade se torna mais desenvolvida e mecanizada, a demanda por atividade física diminui, diminuindo o gasto energético diário (World..., 1990; Grundy, 1998).

Há também evidências sugerindo forte influência genética no desenvolvimento da obesidade, mas seus mecanismos ainda não estão esclarecidos. Acredita-se que esses fatores possam estar relacionados ao consumo e gasto energético. O controle do apetite e o comportamento alimentar também sofrem influência genética. Há indícios de que o componente genético atua sobre o gasto energético, em especial sobre a taxa metabólica basal (TMB) (Baron, 1995; Grundy, 1998), a qual é, determinada principalmente pela quantidade de massa magra (Hill et al., 1993; Jebb, 1997). Além disso, deve-se considerar que há diferenças individuais na suscetibilidade à obesidade
(Blumenkrantz, 1997).

Grundy (1998) destaca em sua revisão que o envelhecimento também está ligado ao ganho de peso, por estar associado a fatores como declínio na TMB em conseqüência da perda de massa muscular, diminuição na prática de atividades físicas e aumento no consumo alimentar.

Um certo número de desordens endócrinas também podem conduzir à obesidade, como por exemplo o hipotireoidismo e problemas no hipotálamo, mas estas causas representam menos de $1 \%$ dos casos de excesso de peso. Outros problemas dessa mesma origem incluem alterações no metabolismo de corticoesteróides, hipogonadismo em homens e ovariectomia em mulheres, e a síndrome do ovário policístico, a qual pode estar relacionada a mudanças na função ovariana ou à hipersensibilidade no eixo hipotálamo-hipófise-adrenal (Baron, 1995; Jebb, 1997).

Problemas psicológicos também estão associados ao ganho de peso, como por exemplo estresse, ansiedade e depressão (Baron, 1995; Jebb, 1997), influenciando principalmente o comportamento alimentar (Stunkard \& Wadden, 1992).

Segundo Jebb (1997), provavelmente a etiologia da
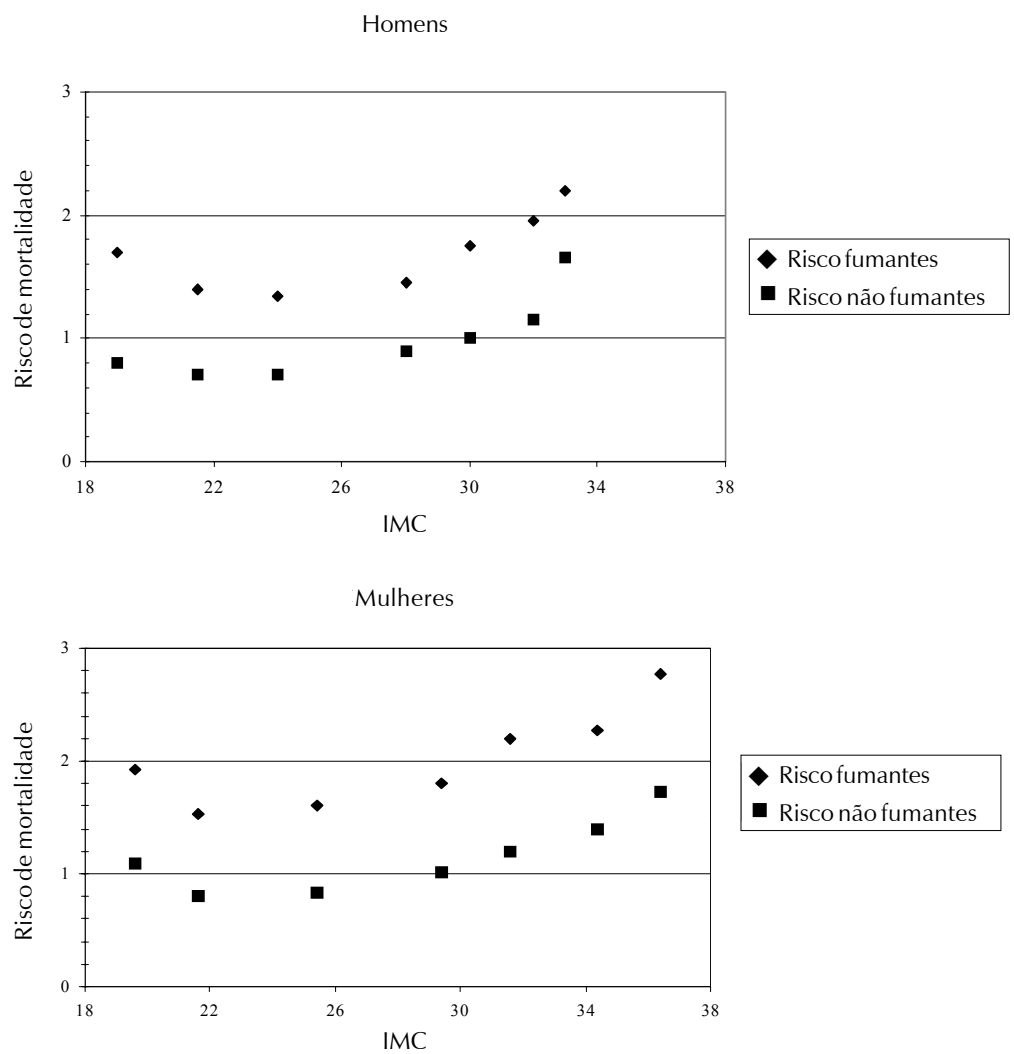

Figura 1. Taxa de mortalidade (média de todas as idades), para homens e mulheres, fumantes e não fumantes, em função do Índice de Massa Corporal (MC).

Fonte: Modificado de World... (1990). 
obesidade é uma das mais complexas. De fato, o seu desenvolvimento possui múltiplas causas e é o resultado de complexas interações entre fatores genéticos, psicológicos, socioeconômicos, culturais e ambientais (Blumenkrantz, 1997).

\section{MORBIDADE E MORTALIDADE ASSOCIADAS À OBESI- DADE}

A obesidade está associada a algumas das mais prevalentes doenças na sociedade moderna. O maior risco é para o desenvolvimento de diabetes mellitus. Quando o Índice de Massa Corporal (IMC) ( $\left.\mathrm{kg} / \mathrm{m}^{2}\right)$ está acima de 35, aumenta o risco de seu desenvolvimento em 93 vezes em mulheres e 42 vezes em homens (Jung, 1997). Os maiores riscos a saúde causados pela obesidade aumentam progressivamente e desproporcionalmente aumento de peso, numa curva conhecida como em forma de "J". Além disso, o risco de mortalidade agrava-se ainda mais para pessoas obesas fumantes (World..., 1990; Baron, 1995; Blumenkrantz, 1997) (Figura 1).

Comparando com pessoas de peso normal, homens com $20 \%$ acima do peso desejável têm $20 \%$ a mais de chance de morrer por todas as causas; possuem o risco duas vezes maior de falecer por diabetes; têm $40 \%$ a mais de chance de desenvolver disfunções na vesícula biliar e $25 \%$ a mais de doenças coronarianas. Em homens com $40 \%$ acima do peso desejável, a mortalidade por todas as causas é $55 \%$ maior, apresentam $70 \%$ a mais de chance de desenvolver doenças coronarianas, e o risco de morte por diabetes é quatro vezes maior do que entre pessoas de peso normal (Blumenkrantz, 1997).

Como pode-se perceber, apesar das doenças coronarianas representarem a maior causa de mortes relacionadas ao excesso de peso, as pessoas obesas freqüentemente desenvolvem outras condições que as predispõem à mortalidade, em especial o diabetes mellitus e doenças do trato digestivo, além das neoplasias (Jung, 1997).

A ocorrência de complicações da obesidade depende não apenas do excesso de peso, mas também da distribuição da gordura corporal, a qual pode estar localizada na região central ou abdominal (conhecida como obesidade em forma de maçã ou andróide) ou na região inferior ou do quadril (conhecida como em forma de pêra ou ginóide). A presença de tecido adiposo intra-abdominal é um fator de risco para distúrbios metabólicos (Grundy, 1998) e é determinada pela relação entre as circunferências da cintura e do quadril (Hauner, 1995).

\section{Diabetes mellitus não-dependente de insulina}

A obesidade, particularmente aquela localizada na região abdominal, pode elevar o risco da ocorrência de Diabetes Mellitus não-dependente de insulina em dez vezes. (Blumenkrantz, 1997). Segundo Jung (1997), em torno de $75 \%$ dos pacientes diabéticos não-dependentes de insulina estão acima do peso desejável. Para aumento de $10 \%$ no peso corporal, há aumento de $2 \mathrm{mg} / \mathrm{dl}$ na glicemia em jejum (Blumenkrantz, 1997). Considerando a distribuição da gordura corporal, Jung (1997) afirma que a circunferência da cintura maior do que $100 \mathrm{~cm}$ pode isoladamente elevar o risco do desenvolvimento de diabetes em 3,5 vezes, mesmo após um controle do IMC.

No desenvolvimento de diabetes, o tecido adiposo atua aumentando a demanda por insulina e, em pacientes obesos, criando resistência à esta, o que ocasiona aumento na glicemia e conseqüente hiperinsulinemia. Contudo, a sensibilidade do tecido adiposo à insulina pode permanecer alta, o que sugere que a lipogênese possa estar favorecida. Em alguns casos, essa resistência pode ser atribuída à diminuição na concentração de receptores de insulina, ou em falha no mecanismo de trânsito celular (Blumenkrantz, 1997).

Há um conjunto de desordens metabólicas e de complicações vasculares decorrentes da obesidade, denominado Síndrome Metabólica ou Síndrome X (Ivkovic-Lazar et al., 1992; Grundy, 1998). Segundo Hauner (1995), os componentes dessa síndrome são caracterizados pela hiperinsulinemia e por várias formas e graus de resistência à insulina, que explicam a relação entre várias disfunções e obesidade. Para Krotkiewski (1994), o aumento da resistência à insulina e a conseqüente hiperinsulinemia em mulheres obesas estão associados ao aumento do tecido adiposo abdominal e as alterações hormonais. A hiperinsulinemia promove inibição da síntese de proteínas específicas transportadoras de testosterona. Conseqüentemente, há aumento da concentração de testosterona livre, o que induz características andrógenas em mulheres, como o acúmulo de gordura na região abdominal. Dessa forma, a diabetes tipo Il é agravada, uma vez que a resistência à insulina e a hiperinsulinemia são fatores predisponentes para o acúmulo de gordura abdominal e para várias outras doenças, como a hipertensão, as doenças cardiovasculares (Defronzo \& Ferrannini, 1991) e as neoplasias (Stoll, 1995).

\section{Hipertensão}

Em jovens adultos de 20 a 45 anos, a prevalência da hipertensão é seis vezes maior em obesos do que em não obesos (Blumenkrantz, 1997). Para cada aumento de 10\% na gordura corporal, há elevação na pressão arterial sistólica de aproximadamente $6,0 \mathrm{mmHg}$ e na diastólica de 4,0 mmHg (Jung, 1997).

A obesidade abdominal está mais associada ao aumento da pressão arterial do que a obesidade localizada 
na região do quadril. Em pacientes obesos, o acúmulo de gordura intra-abdominal resulta aumento da liberação de ácidos graxos livres (AGL) na veia porta, elevando a síntese hepática de triacilgliceróis, aumentando a resistência à insulina e a hiperinsulinemia (Blumenkrantz, 1997). Segundo Defronzo \& Ferrannini (1991), a hipertensão é decorrente da resistência a esse hormônio e da hiperinsulinemia, as quais contribuem para aumento de retenção de sódio pelas células e na atividade do sistema nervoso simpático, distúrbio no transporte iônico da membrana celular e conseqüente aumento da pressão sangüínea.

\section{Doenças cardiovasculares}

As três principais causas de morte no Brasil são o infarto do miocárdio, a insuficiência cardíaca e o acidente vascular cerebral, representando 300 mil mortes anuais ou 820 por dia. O total de mortes no Brasil por doença cardiovascular é de 34,0\% (Sociedade..., 1999b). Krummel (1998) cita os países que apresentam as maiores taxas de mortes por essa causa: Federação Russa (45,2\%), Bulgária $(53,6 \%)$, Inglaterra $(45,4 \%)$, Alemanha $(39,6 \%)$, Noruega (45,0\%), Áustria (40,3\%) e Estados Unidos (38,8\%). Esses são apenas alguns exemplos da verdadeira epidemia mundial que vêm ocorrendo, conhecida pelos cardiologistas como a epidemia da cardiopatia isquêmica (Sociedade..., 1999b).

Para cada $10 \%$ de aumento no peso corporal, há aumento na incidência de doenças coronarianas em aproximadamente $20 \%$, além da elevação no colesterol plasmático em torno de 12 mg/dl (Blumenkrantz, 1997). Isso está relacionado com a dislipidemia na obesidade, representada pela elevação do colesterol total, da lipoproteína de baixa densidade (Low Density Lipoprotein - LDL-colesterol) e dos triglicérides circulantes, e diminuição na lipoproteína de alta densidade (Hight Density Lipoprotein - HDL-colesterol). Esse risco pode se tornar mais acentuado quando o ganho de peso está acompanhado por redução na atividade física e alta ingestão de ácidos graxos saturados (Jung, 1997; Grundy, 1998).

Segundo Defronzo \& Ferrannini (1991), as doenças cardiovasculares têm origem também com a hiperinsulinemia, a qual aumenta a síntese de lipoproteína de muito baixa densidade (Very Low Density Lipoprotein - VLDL-colesterol), conduzindo a hipertrigliceridemia. Com isso ocorre aumento no transporte arterial de colesterol e eleva-se a síntese de lipídeos endógenos. Posteriormente, há aumento na síntese de colágeno nas células da parede vascular e na formação de placas de lipídeos nas artérias associada a diminuição de sua remoção. Assim, haverá predisposição à formação do ateroma, elevando a probabilidade de ocorrência de problemas cardiovasculares.

A localização do tecido adiposo na região abdominal também predispõe a problemas cardiovasculares. Uma forma simples para medir o grau de adiposidade intra-abdominal consiste na razão entre as circunferências da cintura e do quadril: para homens, o risco de desenvolver esse tipo de doença aumenta quando a relação cintura/quadril é acima de 1,0 e, para mulheres, quando essa relação é acima de 0,8. Quando isso ocorre em mulheres pré-menopausa, há diminuição progressiva na ligação entre os hormônios sexuais e a globulina, aumentando a concentração de testosterona livre, o que eleva a atividade androgênica e as concentrações de AGL. Em mulheres pós-menopausa a deficiência de estrógeno também contribui para a distribuição de tecido adiposo característico do sexo masculino, e a perda da função ovariana está associada com o desenvolvimento de problemas aterogênicos (Gaspard, 1995).

\section{Neoplasias}

Homens com sobrepeso têm mortalidade significantemente maior por câncer colorectal e de próstata: homens cujo peso é cerca de $130 \%$ maior do que o peso médio para o seu biótipo têm 2,5 mais chances de morrer por câncer de próstata que indivíduos normais (Blumenkrantz, 1997).

Mulheres acima do peso também têm maiores chances de desenvolverem câncer de colo uterino, ovário e mama (Blumenkrantz, 1997; Jung, 1997). Segundo Stoll (1995), além da contribuição do excesso de peso para o aumento na ocorrência de neoplasias, a concentração do tecido adiposo na região abdominal aliada à síndrome de resistência a insulina em obesas, elevam o risco de câncer de mama.

\section{Disfunções endócrinas}

Mulheres obesas, em especial as que apresentam obesidade abdominal, desenvolvem irregularidades no ciclo menstrual e amenorréias e apresentam mais problemas durante a gravidez, como a síndrome hipertensiva e a toxemia (Givens, 1992; Blumenkrantz, 1997). Garotas obesas geralmente possuem a menarca em idades mais novas do que jovens com peso normal, já que a menstruação é provavelmente iniciada quando o peso corporal atinge certa massa corporal crítica (Blumenkrantz, 1997). O aumento do tecido adiposo intra-abdominal, da concentração de testosterona livre e da resistência à insulina também pode implicar desenvolvimento da síndrome do ovário policístico (Jung, 1997). 


\section{Disfunção da vesícula biliar}

A formação de cálculo na vesícula biliar é a forma mais comum de doença do trato digestivo em obesos. Mulheres obesas entre 20 e 30 anos apresentam um risco seis vezes maior no desenvolvimento de disfunção na vesícula biliar do que mulheres com o peso normal. Com a idade, em torno de 60 anos aproximadamente, um terço das mulheres obesas devem apresentar essa doença. A associação entre obesidade e cálculo na vesícula biliar pode relacionar-se com dois fatores: aumento no colesterol circulante quando os estoques de tecido adiposo são mobilizados; e aumento na taxa em que o colesterol é excretado na bile. A formação do cálculo na vesícula biliar depende da precipitação do colesterol de bile saturada. Para cada $1 \mathrm{~kg}$ de gordura corporal, aproximadamente 20

Quadro 1. Morbidade em pessoas obesas. $\mathrm{mg} / \mathrm{dl}$ de colesterol é sintetizado e, em obesos, a bile é muito mais saturada com colesterol (Blumenkrantz, 1997; Jung, 1997).

\section{Problemas pulmonares}

Há uma série de disfunções pulmonares em indivíduos obesos, como por exemplo a chamada síndrome Pickwickian ou síndrome da obesidade-hipoventilação, caracterizada por sonolência e redução da ventilação. Há queda uniforme no volume de reserva expiratório e na capacidade vital (Kopelman, 1992). O aumento na quantidade de gordura acumulada na região peitoral e abdominal limita os movimentos respiratórios e diminui o volume pulmonar (Jung, 1997). Na medida em que o indivíduo se torna mais obeso, ocorre sobrecarga muscular para a ventilação, resultando em disfunção da musculatura

\begin{tabular}{|c|c|}
\hline Cardiovasculares & Região peitoral \\
\hline Hipertensão & Câncer de mama \\
\hline Doenças coronarianas & Ginecomastia \\
\hline Acidente vascular cerebral & \\
\hline Veias varicosais & Utero \\
\hline Trombose venosa profunda & Câncer endometrial \\
\hline & Câncer cervical \\
\hline \multicolumn{2}{|l|}{ Respiratórias } \\
\hline Falta de ar & Urológico \\
\hline Apnéia durante o sono & Câncer de próstata \\
\hline Síndrome hipoventilação & Incontinência urinária \\
\hline Gastrintestinais & Pele \\
\hline Hérnia de hiato & Micoses \\
\hline Cálculo na vesícula biliar & Linfoedemas \\
\hline Cirrose e esteatose hepática & Celulites \\
\hline Hemorróida & Acantose \\
\hline Câncer colorectal & Ortopédicas \\
\hline Metabólica & Osteoartrites \\
\hline Hiperlipidemia & Gota \\
\hline Resistência à insulina & Endócrinas \\
\hline Diabetes mellitus & Redução no GH \\
\hline Síndrome do ovário policístico & Redução na resposta à prolactina \\
\hline Hiperandrogenização & Respostas hiperdinâmicas do $\mathrm{ACTH}$ ao $\mathrm{CRH}$ \\
\hline Irregularidades menstruais & Aumento do cortisol livre na urina \\
\hline \multicolumn{2}{|r|}{ Alterações nos hormônios sexuais } \\
\hline Bloqueio nervoso & Gravidez \\
\hline Renal & Complicações obstétricas \\
\hline \multirow[t]{3}{*}{ Proteinúria } & Operação por cesariana \\
\hline & Bebês muito grandes \\
\hline & Defeitos no tubo neural \\
\hline
\end{tabular}

$\mathrm{GH}=$ hormônio do crescimento; $\mathrm{ACTH}=$ hormônio adrenocorticotrófico; $\mathrm{CRH}=$ hormônio liberador de $\mathrm{ACTH}$ Fonte: Modificado de Jung (1997). 
respiratória (Blumenkrantz, 1997).

Artrites
A relação entre artrite e obesidade consiste em problema mecânico e não metabólico (Blumenkrantz, 1997). O excesso de peso facilita a ocorrência de traumas, principalmente nas articulações, como a osteoartrite no

Quadro 2. Benefícios da diminuição de $10 \mathrm{~kg}$ no peso corporal.

\begin{tabular}{ll}
\hline Mortalidade & Queda de $20-25 \%$ na mortalidade total \\
& Declínio de $30-40 \%$ nas mortes por diabetes \\
& $40-50 \%$ de diminuição nas mortes por neoplasias da obesidade \\
Pressão arterial & Queda de $10 \mathrm{mmHg}$ na pressão sistólica \\
& Diminuição de $20 \mathrm{mmHg}$ na pressão diastólica \\
Angina & Redução nos sintomas em $91 \%$ \\
& Aumento de $33 \%$ na tolerância ao exercício \\
Lipídeos & Diminuição de $10 \%$ no colesterol total \\
& Declínio de $15 \%$ no LDL-colesterol \\
& Diminuição de $30 \%$ nos triglicérides circulantes \\
& Aumento em $8 \%$ no HDL-colesterol \\
Diabetes & Redução no risco de desenvolvimento de diabetes maior do que $50 \%$ \\
& Diminuição de $30-50 \%$ na glicemia de jejum \\
& Declínio de $15 \%$ em HbA1c \\
\hline
\end{tabular}

Fonte: Modificado de Jung (1997)

joelho. Contudo, o excesso de tecido adiposo pode atuar no metabolismo esquelético, devido à alteração no metabolismo de estrógeno (Rössner, 1989; Bollet, 1992).

A obesidade ainda pode ser associada a uma série de desordens, como problemas no trato digestivo (problemas no fígado, esofagite), tromboembolias, diminuição na capacidade cardíaca e problemas de pele, maior incidência de complicações cirúrgicas e obstétricas, e mais suscetibilidade a acidentes. Apesar da obesidade não estar associada ao aumento de risco em problemas psiquiátricos, geralmente esses pacientes têm incidência maior de problemas psicológicos e de discriminação social (Baron, 1995) (Quadro 1).

\section{TRATAMENTOS PARA A REDUÇÃO DA OBESIDADE}

Muitos estudos demonstram que a redução da quantidade de massa corporal, em especial de gordura, melhora a qualidade de vida e diminui a morbidade e a mortalidade de pacientes obesos. Jung (1997) ilustra em sua revisão os benefícios da perda de $10 \mathrm{~kg}$ de peso para a saúde desses indivíduos (Quadro 2).

Contudo, deve-se questionar a relação entre redução de peso e de gordura corporal, os quais, na maioria das vezes, são utilizados como sinônimos de forma errônea. É possível reduzir a gordura corporal, sem diminuir o peso quando, por exemplo, ocorre ganho de massa muscular. O aumento de massa muscular pode ser superior ao peso de gordura reduzido, levando ao aumento no peso corporal total. Assim, a ênfase no tratamento da obesidade deve ser na redução da gordura corporal, já que apenas a perda de gordura promoverá benefícios a saúde.

Para conseguir essa diminuição da massa gordurosa é necessário um balanço energético negativo, condição na qual o gasto supera o consumo de energia, pois os estoques de energia do organismo são consumidos para sustentar processos metabólicos, levando a perda de peso. Para que isso ocorra, deve-se considerar três componentes da equação de balanço energético, definida como: Gasto energético $=\mathrm{TMB}+\mathrm{E}_{\text {exercício físico }}+\mathrm{ETA}$ na qual a TMB é a taxa metabólica basal, $\mathrm{E}_{\text {exercício físico }}$ corresponde à energia gasta nas atividades físicas e ETA é o efeito térmico do alimento (Hill et al., 1993).

A taxa metabólica basal depende da idade, sendo determinada principalmente pelo total de massa magra (Hill et al., 1993). A energia gasta durante as atividades físicas depende da intensidade e da duração desta, e corresponde ao maior efeito sobre o consumo de energia humana, já que é possível gerar taxas metabólicas dez vezes superiores aos valores de repouso durante um exercício com participação de grandes grupos musculares. O efeito térmico do alimento, também conhecido por termogênese de indução dietética, deve-se principalmente aos processos de digestão, absorção e assimilação dos nutrientes, e representa, para uma pessoa ativa, apenas uma pequena parte do gasto energético diário (McCardle et al., 1992). 
Entretanto, algumas considerações devem ser feitas: ao estimar o gasto calórico do exercício físico e o efeito térmico do alimento, estaremos somando, durante o espaço de tempo que dura esta atividade, o gasto basal mais o gasto da atividade ou da alimentação. Assim, deve--se debitar do tempo total da TMB esse período de exercício e de alimentação.

A literatura é extensa quanto aos diferentes tratamentos para a obesidade, porém ainda não foi possível alcançar uma conclusão definitiva (Cowburn et al., 1997).

\section{Dieta}

Muitos tratamentos para a obesidade envolvem a restrição da ingestão energética total, uma das formas de alcançar o déficit energético e reduzir o peso corporal (Raccete et al., 1995a). Segundo Wirth (1995). A dieta para redução de peso deve limitar a ingestão total energética. Duas estratégias comumente utilizadas são o uso das Very Low Calorie Diets (VLCD) com consumo energético menor de $800 \mathrm{kcal} / \mathrm{dia}$, e as restrições energéticas moderadas, com consumo de $1200 \mathrm{kcal} / \mathrm{dia}$ ou mais (Cowburn et al., 1997). No caso das VLCD, a perda de peso é mais rápida, em torno de 1,5-2,5 kg por semana, enquanto na restrição moderada a diminuição é de 0,5-0,6 kg/semana. Contudo, o maior problema das VLCD é a dificuldade da manutenção do peso perdido após o término da dieta (Cowburn et al., 1997). Segundo American... (1983), dietas que restringem severamente o consumo energético, bem como jejuns prolongados, são cientificamente indesejáveis e perigosos para a saúde, resultando em perdas de grandes quantidades de água, eletrólitos, minerais, glicogênio e outros tecidos isentos de gordura, com mínima redução de massa adiposa.

Ilarde \& Tuck (1994) acreditam que não só o total energético deve ser controlado para pacientes que apresentam a síndrome metabólica, afirmando que a dieta deve conter alta porcentagem de energia derivada de carboidratos e baixa de lipídeos, além de controle na quantidade de colesterol na dieta, a fim de reduzir as chances de ocorrência de problemas cardiovasculares. Do mesmo modo, Lean et al. (1989) defendem dietas ricas em carboidratos e pobre em lipídeos, para prevenção de obesidade e manutenção do peso adequado. De fato, a composição da dieta parece influenciar a redução de peso: dietas ricas em lipídeos podem criar balanço positivo deste nutriente no organismo, ou seja, seu consumo é maior do que a sua oxidação. Isso ocorre porque o aumento na ingestão de lipídeos não estimula a sua oxidação, o que ocorre com carboidratos e proteínas, e porque a eficiência do organismo em estocar os lipídeos da dieta como reservas de gordura é muito alta, superior a 95\% (Hill et al., 1993; Swinburn \& Ravussin, 1993).

A quantidade adequada de fibras alimentares tem importante função na dieta para redução de obesidade, tais como: redução na ingestão energética; aumento no tempo de esvaziamento gástrico; diminuição na secreção de insulina; aumento na sensação de saciedade; redução na digestibilidade; redução no gasto energético e aumento na excreção fecal de energia (Rössner, 1992). Dietas ricas em fibras também contribuem para a minimização dos problemas de doenças cardiovasculares, devido à redução do colesterol plasmáticos e da LDL. As fibras provavelmente interferem no metabolismo dos esteróides, que começa no trato gastrintestinal. Essa interferência ocorre por serem as fibras pouca digeridas e absorvidas pelo organismo humano, aumentando a excreção fecal de colesterol presente nos ácidos biliares (O’Dea, 1991; Truswell \& Beynen, 1992).

Sujeitos submetidos a dietas hipocalóricas sofrem mudanças adaptativas que ocorrem em resposta à limitação no consumo energético para permitir o prolongamento da vida do indivíduo. À medida que o consumo energético é restrito, o gasto energético diminui, levando à redução da perda de peso com o tempo, em conseqüência de mudanças na composição corporal (Shetty, 1990). São responsáveis por essas modificações a redução na taxa metabólica basal (TMB) (Ballor et al., 1996), a diminuição na massa magra (Ravussin et al., 1985); e a redução no custo energético do movimento pela queda nas dimensões corporais (Froidevaux et al., 1993), que diminui a oxidação de gorduras (Ballor et al., 1996).

A American... (1983) recomenda que a perda de peso deve ser resultado da máxima redução de gordura corporal e de mínima perda de massa magra, representando sucesso na manutenção do peso perdido, poucos riscos de desnutrição e de complicações médicas. Apenas com dieta hipocalórica, isso é pouco provável: o estudo realizado por Hill et al. (1987) demonstrou que indivíduos sedentários consumindo $800 \mathrm{kcal} /$ dia durante 5 semanas perderam $8,2 \pm 0,5 \%$ de seu peso corporal inicial, sendo que $57 \pm$ $4 \%$ desse total perdido foi de gordura e $43 \pm 4 \%$ foi de massa magra.

A redução na TMB é um dos mais constantes resultados observados durante experimentos com déficit energético. Inicialmente (nas primeiras duas ou três semanas), a redução na TMB não pode ser atribuída à mudanças na composição corporal, e sim ao aumento na eficiência do metabolismo dos tecidos ativos. Com o prosseguimento da restrição, esta queda na TMB ocorre devido a perda destes tecidos ativos, em especial dos tecidos magros. Muitos mecanismos fisiológicos operam na diminuição da atividade metabólica, tais como diminuição da atividade do sistema nervoso simpático, mudanças periféricas no metabolismo tireoidiano, redução na secreção de insulina, mudanças na secreção de glucagon, hormônio de crescimento (Grow Hormony - GH) e glucocorticóides. Essas mudanças promovem a mobilização de substratos endógenos, conduzindo a maior circulação de ácidos graxos e corpos cetônicos, além de aumentar o catabolismo de proteínas, atuando sobre a diminuição do gasto energético (Shetty, 1990). 
Tais alterações fisiológicas em resposta ao déficit energético podem trazer conseqüências para o organismo: redução de proteína muscular, ou mesmo a diminuição de proteína hepática (Walberg, 1989). Alterações mais sérias podem ocorrer na proteína das fibras musculares cardíacas: exames dos corações de 17 pacientes que morreram após se submeterem a grande redução de peso (mais de 30\% do peso inicial) revelaram redução da massa cardíaca e atrofia das fibras musculares desse órgão (Van Ittalie \& Yang, 1984). Não há dúvidas que restrições energéticas alteram a massa magra, sendo esses efeitos mais intensos quanto maior for o déficit energético (Sweeney et al., 1993) e mais prolongado for o período de tempo de restrição pelo qual o indivíduo foi submetido.

Um tratamento que previna ou mesmo que diminua esse efeito oferece vantagem frente aos demais (Racette et al., 1995a). A inclusão de exercícios físicos pode ser favorável nesse sentido.

\section{Exercícios físicos}

O exercício físico regular resulta benefícios para o organismo, como melhora na capacidade cardiovascular e respiratória, diminuição na pressão arterial em hipertensos, melhora na tolerância à glicose e na ação da insulina (Dengel et al., 1998). O exercício regular está associado com diminuição da mortalidade em geral e a longo prazo (Baron, 1995).

Segundo Blair (1993), indivíduos fisicamente ativos e com excesso de peso apresentam menor morbidade e mortalidade que aqueles sedentários, pelo aumento na sensibilidade à insulina e melhora na tolerância à glicose, e no metabolismo lipídico (Oshida et al., 1989). Sabe-se que um dos transportadores de glicose na células musculares e adipócitos (Glut 4) é regulado pela atividade contráctil dos músculos, e insulina. Esses dois estímulos translocam essa proteína dos compartimentos intracelulares para a membrana plasmática, para receber e transportar a glicose para dentro da célula (Hardin et al., 1995).

O exercício contribui para redução de peso através da criação de balanço energético negativo (Melby et al., 1993). Contudo, atingir altos gastos energéticos durante a atividade física requer a capacidade do indivíduo para se exercitar por longos períodos em altas intensidades, o que é possível para pessoas treinadas. Por esse motivo, a dieta isolada é mais eficiente para produzir déficit energético do que o exercício físico (Björntorp, 1995; Saris, 1995), o que fez alguns autores desconsiderarem a importância deste na obesidade (Garrow, 1995). De fato, esperar que um sedentário no início de um programa de exercícios alcance o mesmo gasto energético induzido pela dieta hipocalórica é irreal, mas não significa que o exercício desempenhe apenas um impacto marginal na perda de peso a longo prazo (Melby et al., 1998). O aumento da prática de atividade física significa uma perturbação no equilíbrio do balanço energético e de macronutrientes. O treinamento induz à mudanças adaptativas: o aumento na capacidade de se exercitar em altas intensidades por períodos maiores, e o aumento da oxidação de gorduras, que promove sua perda gradual e mantém o peso perdido alcançado inicialmente com a dieta hipocalórica (Melby et al., 1998). De fato, o estudo recente de Nicklas et al. (1997) demonstra que o exercício aeróbio combinado à dieta previne o declínio na resposta lipolítica e na oxidação de gorduras que ocorre em obesos submetidos apenas à dieta. Outro aspecto positivo da combinação dieta e exercícios é quanto à manutenção do peso perdido; Miller et al. (1997) concluíram em sua meta-análise que, após um ano do final do tratamento, a manutenção do peso perdido em dois grupos de obesos (submetidos a dieta isolada ou combinada com exercícios por 15 semanas) é maior no grupo exercitado.

O exercício físico é uma forma de tratamento de obesidade que eleva o gasto energético e minimiza os efeitos negativos da restrição energética, pois é capaz de reverter a queda na TMB (Molé et al., 1989). Segundo Forbes (1992), o exercício aeróbio preserva a massa magra, e ajuda a manter a TMB durante um programa de redução de peso, desde que não haja um déficit energético muito grande. Além disso, a combinação de dieta e de exercício de intensidade moderada geralmente promove uma maior perda de peso do que a dieta isolada. O exercício combinado à restrição energética promove redução no peso corporal, maximizando a perda de gordura e minimizando a perda de massa magra (Hill et al., 1987; Kempen et al., 1995; Racette et al., 1995b; Cowburn et al., 1997). As alterações de composição corporal induzidas pelo treinamento físico também dependem do tipo, da intensidade e duração deste, sendo que os efeitos de manutenção de massa magra são freqüentemente vistos quando se aplica treinamentos de força (Ballor et al., 1988; Ross et al., 1995; Geliebter et al., 1997).

No entanto, outros estudos mostram que, apesar dos numerosos benefícios da atividade física regular, esta não trouxe mudanças significativas no peso, na composição corporal e na TMB (Katch et al., 1988, Donnely et al., 1991). Mas Racette et al. (1995a) observaram que o exercício aeróbio promoveu perda de massa gorda e manteve o gasto energético total diário, por aumentar o tempo de atividade física diária, e concluem que esse tipo de exercício em associação ao controle nutricional é um componente importante no tratamento da obesidade.

Os estudos realizados por King et al. (1994) também apontaram que o exercício pode apresentar um efeito anoréxico - uma temporária supressão da fome acompanhado por adiamento da alimentação após a sessão de exercício.

Racette et al. (1995b) referem a maior adesão à dieta entre aqueles que associam a atividade física. Ao estudar 30 mulheres obesas por 12 semanas, divididas em dois grupos: aquele que pratica exercício aeróbio e faz dieta e o que faz exclusivamente dieta, os autores notaram que o grupo exercitado seguiu a dieta de forma mais 
efetiva.

Tendo em vista os inúmeros benefícios que o exercício físico regular induz, este deve ser parte das estratégias de redução de gordura corporal.

\section{Medicamentos}

O tratamento medicamentoso para redução da obesidade utiliza as seguintes drogas: anfetaminas, fenfluraminas, fenterminas, dietilpropriona, mazindol, pemolina, fenilpropanolamina e os anti-depressivos, fluoxitena e sertralina, entre outras. Há muitas controvérsias sobre a utilização destes, pelos escassos estudos sobre seus efeitos a longo prazo. Os remédios criam uma expectativa de cura para a obesidade e as pessoas comumente voltam a engordar com a suspensão do medicamento. Os efeitos colaterais normalmente observados incluem sonolência, nervosismo e distúrbios no trato gastrintestinal (Baron, 1995).

\section{Mudança comportamental}

Tendo em vista fatores cognitivos e emocionais associados ao aumento do consumo de alimentos (Foreyt \& Goodrick, 1993), a mudança comportamental tem sido usada no tratamento da obesidade. O auto-monitoramento faz parte do programa de mudança comportamental e consiste em auto-observação dos fatos, sentimentos, pensamentos e atitudes que ocorrem antes, durante e após as tentativas de manter um comportamento prudente ao alimentar-se e na prática de exercícios físicos (Foreyt \& Goodrick, 1993). Segundo Atckinson et al. (1992), os componentes de um programa de mudança comportamental incluem: educação sobre a etiologia e a fisiopatologia da obesidade; educação alimentar, nutricional e novas técnicas dietéticas; educação através da fisiologia do exercício, estratégias, técnicas e monitoramento da atividade física; conhecimento de estratégias para evitar o ganho de gordura novamente; apoio familiar, social e acompanhamento de uma equipe multidisciplinar de profissionais de saúde.

\section{PREVENÇÃO}

A importância da redução da obesidade para a saúde pública, o interesse social e os investimentos econômicos em alimentação e na qualidade de vida de pessoas obesas indicam que, estudos rigorosos sobre a prevenção e o tratamento da obesidade são essenciais (Hyman et al., 1993). Algumas medidas preventivas citadas por Gill (1997) em sua revisão consideram a interação entre estratégias administrativas e a comunidade, tais como: alterações na estrutura urbana, como construção de calçadas seguras e de ciclovias; melhora nos designs das construções a fim de facilitar o uso de escadas; legislação e regulação dos rótulos dos produtos alimentícios; subsídios para produtores de alimentos com pouca densidade calórica (em especial, frutas e vegetais); incentivos fiscais para empresas que incentivem a prática de atividades físicas dos empregados; consultoria nutricional para refeições escolares e empresariais, entre outras. De fato, intervir de maneira preventiva sobre a obesidade tende a ser mais fácil, menos caro e potencialmente mais efetivo.

\section{AGRADECIMENTOS}

À bibliotecária Maria Lúcia F. Ferraz do Centro de Informação e Referência (CIR) em Saúde Pública da Faculdade de Saúde Pública da USP, pelas informações técnicas indispensáveis à execução desse trabalho.

À Letícia A. Sawada, médica e mestranda da EEFE-USP e a todos da equipe do Laboratório de Nutrição e Metabolismo Aplicado à Atividade Motora da EEFE-USP, pelo apoio, colaboração e competência em todas atividades por nós realizadas.

\section{REFERÊNCIAS BIBLIOGRÁFICAS}

AMERICAN COLLEGE OF SPORTS MEDICINE. Position stand: proper and improper weight loss programs. Medicine and Science in Sports and Exercise, Baltimore, v.15, p.9-13, 1983.

ATKINSON, R.L., FUCHS, A., PASTORS, J.G., SAUNDERS, J.T. Combination of very-low-calorie diet and behavior modification in the treatment of obesity. American Journal of Clinical Nutrition, Bethesda, v.56, n.1, p.199S- 202S, 1992. Supplement.

BALLOR, D.L., KATCH, V.L., BECQUE, M.D., MARKS, C.R. Resistance weight training during caloric restriction enhances lean body weight maintenance. American Journal of Clinical Nutrition, Bethesda, v.47, n.1, p.19-25, 1988.

BALLOR, D.L., HARVEY-BERINO, J.R., ADES, P.A., CRYAN, J., CALLES-ESCANDON, J. Contrasting effects of resistance and aerobic training on body composition and metabolism after diet-induced weight loss. Metabolism, London, v.45, n.2, p.179-183, 1996.

BARON, R. Understanding obesity and weight loss [online]. 1995 [citado em 28/8/97]. Available from WWW: <URL: http://www-med.stanford.edu;school;DGIM;Teaching; +Modules;obesity.html>.

BJÖRNTORP, P. Evolution of the understanding of the role of exercise in obesity and its complications. International Journal of Obesity and Related Metabolic Disorders, Hampshire, v.19, n.4, p.1S-4S, 1995. Supplement.

BLAIR, S.N. Evidence for success of exercise in weight loss and control. Annal of Internal Medicine, Philadelphia, v.119, n.7 (Pt 2), p.702-706, 1993.

BLUMENKRANTZ, M. Obesity: the world's metabolic disorder [online]. Beverly Hills, 1997. [citado em 28/8/97]. Available from WWW: <URL: http:// www.quantumhcp.com, obesity.htm $>$.

BOLLET, A.J. Obesity and musculoskeletal disease. In: 
BJÖRNTORP, P., BRODOFF, B.N. Obesity. Philadelphia : J.B. Lippincott, 1992. p.563-567.

BRAY, G. An approach to the classification and evaluation of obesity. In: BJÖRNTORP, P., BRODOFF, B.N. Obesity, Philadelphia : J.B. Lippincott, 1992. p.294-308.

COWBURN, G., HILLSDON, M., HANKEY, C.R. Obesity management by life-style strategies. British Medical Bulletin, London, v.53, n.2, p.389-408, 1997.

DEFRONZO, R.A., FERRANNINI, E. Insulin resistance: a multifaceted syndrome responsible for NIDDM, obesity, hypertension, dyslipidemia, and atherosclerotic cardiovascular disease. Diabetes Care, New York, v.14, n.3, p.173-194, 1991.

DENGEL, D.R., HAGBERG, J.M., PRATLEY, R.E., ROGUS, E.M., GOLDBERG, A.P. Improvements in blood pressure, glucose metabolism, and lipoprotein lipids after aerobic exercise plus weight loss in obese, hypertensive middle-aged men. Metabolism, London, v.47, n.9, p.1075-1082, 1998.

DONNELLY, J.E., PRONK, N.P., JACOBSEN, D.J., PRONK, S.J., JAKICIC, J.M. Effects of a very-low-calorie diet and physical--training regimens on body composition and resting metabolic rate in obese females. American Journal of Clinical Nutrition, Bethesda, v.54, n.1, p.56-61, 1991.

DYER, R.G. Traditional treatment of obesity: does it work? Baillieres Clinical Endocrinology and Metabolism, London, v.8, n.3, p.661-688, 1994.

FORBES, G. B. Exercise and lean weight: the influence of body weight. Nutrition Reviews, Washington DC, v.50, n.6, p.157-161, 1992.

FOREYT, J.P., GOODRICK, G.K. Evidence for success of behavior modification in weight loss and control. Annals of Internal Medicine, Philadelphia, v.119, n.7 (Pt 2), p.698-701, 1993.

FRANCISCHI, R.P., KLOPFER, M., PEREIRA, L.O., CAMPOS, P.L., SAWADA, L.A., SANTOS, R., VIEIRA, P., LANCHA JR, A.H. Efeito da intensidade da atividade física e da dieta hipocalórica sobre consumo alimentar, a composição corporal e a colesterolemia em mulheres obesas. Revista Brasileira de Nutrição Clínica, Porto Alegre, v.14, n.1, p.1-8, 1999.

FROIDEVAUX, F., SCHUTZ, Y., CHRISTIN, L., JÉQUIER, E. Energy expenditure in obese women before and during weight loss, after refeeding, and in the weight-relapse period. American Journal of Clinical Nutrition, Bethesda, v.57, n.1, p.35-42, 1993.

GARROW, J.S. Exercise in the treatment of obesity: a marginal contribution. International Journal of Obesity and Related Metabolic Disorders, Hampshire, v.19, n.4, p.126S-129S, 1995. Supplement.

GASPARD, U.J. Postmenopausal changes of lipid and glucose metabolism: a review of their main aspects. Maturitas, Limerick, v.21, n.3, p.171-178, 1995.

GELIEBTER, A., MAHER, M.M., GERACE, L., GUTIN, B., HEYMSFIELD, S.B., HASHIM, S.A. Effects of strength or aerobic training on body composition, resting metabolic rate, and peak oxygen consumption in obese dieting subjects. American Journal of Clinical Nutrition, Bethesda, v.66, n.3, p.557-563, 1997.
GILL, T.P. Key issues in the prevention of obesity. British Medical Bulletin, London, v.53, n.2, p.359-388, 1997.

GIVENS, J.R. Reproductive and hormonal alterations in obesity. In: BJÖRNTORP, P., BRODOFF, B. N. Obesity. Philadelphia : J.B. Lippincott, 1992. p.540-549.

GRUNDY, S.M. Multifactorial causation of obesity: implications for prevention. American Journal of Clinical Nutrition, Bethesda, v.67, n.3, p.563S-572S, 1998. Supplement.

HARDIN, D.S., AZZARELLI, B., EDUARDS, J., WIGGLESWORTH, J., MAIANU, L., BRECHTEL, G., JOHNSON, A., BARON, A., GARVEY, W.T. Mechanisms of enhanced insulin sensitivity in endurance-trained athletes: effects on blood flow and differential expression of GLUT 4 in skeletal muscles. Journal of Clinical Endocrinology and Metabolism, Bethesda, v.80, n.8, p.2437-2446, 1995.

HAUNER, H. Abdominal obesity and coronary heart disease: pathophisiology and clinical significance. Herz, Munchen, v.20, n.1, p.47-55, 1995.

HILL, J.O., SPARLING, P.B., SHIELDS, T.W., HELLER, P.A. Effects of exercise and food restriction on body composition and metabolic rate in obese women. American Journal of Clinical Nutrition, Bethesda, v.46, n.4, p.622-630, 1987.

HILL, J.O., DROUGAS, H., PETERS, J.C. Obesity treatment: can diet composition play a role? Annals of Internal Medicine, Philadelphia, v.119, n.7 (Pt 2), p.694-697, 1993.

HYMAN, F.N., SEMPOS, E., SALTSMAN, J., GLISMANN, W.H. Evidence for sucess of caloric restrition in weight loss and control-summary of data from industry. Annals of Internal Medicine, Philadelphia, v.119, n.7 (Pt 2), p.681-687, 1993.

ILARDE, A., TUCK, M. Treatment of non-insulin-dependent diabetes mellitus and its complications: a state of the art review. Drugs and Aging, Auckland, v.4, n.6, p.470-491, 1994.

IVKOVIC-LAZAR, T., LEPSANOVIC, L., BABIC, L., STOKIC, E., TESIC, D., MEDIC-STOJANOSKA, M. The metabolic X syndrome: 4 case reports. Medicinski Pregled, Novi Sad, v.45, n.5/6, p.210-214, 1992.

JEBB, S.A. Aetiology of obesity. British Medical Bulletin, London, v.53, n.2, p.264-285, 1997.

JUNG, R. Obesity as a disease. British Medical Bulletin, London, v.53, n.2, p.307-321, 1997.

KATCH, V., BECQUE, D., MARKS, C., MOOREHEAD, C., ROCCHINI, A. Basal metabolism of obese adolescents: inconsistent diet and exercise effects. American Journal of Clinical Nutrition, Bethesda, v.48, n.3, p.565-569, 1988.

KEMPEN, K.P.G., SARIS, W.H.M., WESTERTERP, K.R. Energy balance during an-8wk energy-restricted diet with and without exercise in obese women. American Journal of Clinical Nutrition, Bethesda, v.62, n.4, p.722-729, 1995.

KING, N.A., BURLEY, V.J., BLUNDELL, J.E. Exercise-induced suppression of appetite: effects on food intake and implications for energy balance. European Journal of Clinical Nutrition, Hampshire, v.48, n.10, p.715-724, 1994.

KOPELMAN, P.G. Altered respiratory function in obesity: sleep-disordered breathing and the Pickwickian syndrome. 
In: BJÖRNTORP, P., BRODOFF, B.N. Obesity. Philadelphia : J.B. Lippincott, 1992. p.568-575.

KROTKIEWSKI, M. Role of muscle morphology in the development of insulin resistance and metabolic syndrome. Presse Medicale, Paris, v.23, n.30, p.1393-1399, 1994.

KRUMMEL, D. Nutrição na doença cardiovascular. In: MAHAN, L.K., ESCOTT-STUMP, S. Krause: alimentos, nutrição e dietoterapia. 9.ed. São Paulo : Roca, 1998. p.525-68.

LEAN, M.E.J., JAMES, W.P.T., GARTHWAITE, P.H. Obesity without overeating? Reduced diet-induced thermogenesis in post-obese women, dependent on carbohydrate and not fat intake. In: BJÖRNTORP, P., RÖSSNER, S. Obesity in Europe 88: proceedings of the $1^{\text {st }}$ european congress on obesity. London: John Libbey, 1989. p.29-41.

MCCARDLE, W.D., KATCH, F.I., KATCH, V.L. Fisiologia do exercício: energia, nutrição e desempenho humano. 3.ed. Rio de Janeiro: Guanabara Koogan, 1992. p.102-112.

MELBY, C.L., SCHOLL, C., EDWARDS, G., BULLOUGH, R. Effect of acute resistance exercise on post exercise energy expenditure and resting metabolic rate. Journal of Applied Physiology, Washington DC, v.75, n.4, p.1847-1853, 1993.

MELBY, C.L., COMMERFORD, S.R., HILL, J.O. Exercise, macronutrient balance, and weight control. In: LAMB, D.R., MURRAY, R. Perspectives in exercise science and sports medicine. Carmel: Cooper Publishing Group, 1998. v.11: p.1-60.

MILLER, W.C., KOCEJA, D.M., HAMILTON, E.J. A meta-analysis of the past 25 years of weight loss research using diet, exercise or diet plus exercise intervention. International Journal of Obesity and Related Metabolic Disorders, Hampshire, v.21, p.941-947, 1997.

MOLÉ, P.A., STERN, J.S., SCHULTZ, C.L., BERNAUER, E.M., HOLCOMB, B.J. Exercise reverses depressed metabolic rate produced by severe caloric restriction. Medicine and Science in Sports and Exercise, Baltimore, v.21, n.1, p.29-33, 1989.

MONTEIRO, C.A., MONDINI, L., SOUZA, A.L.M., POPKIN, B.M. Da desnutrição para a obesidade: a transição nutricional no Brasil. In: MONTEIRO, C.A. Velhos e novos males da saúde no Brasil: a evolução do país e de suas doenças. São Paulo : Hucitec, 1995. p.247-255.

NICKLAS, B.J., ROGUS, E.M., GOLDBERG, A.P. Exercise blunts declines in lipolysis and fat oxidation after dietary-induced weight loss in obese older women. American Journal of Physiology, Bethesda, v.273, n.1 (Pt 1), p.E149-E155, 1997.

O'DEA K. Westernization and non-insulin-dependent diabetes in Australian Aborigines. Ethnicity and Disease, Atlanta GA, v.1, n.2, p.171-187, 1991.

OSHIDA, Y., YAMANOUCHI, K., HAYAMIZU, S., SATO, Y. Long- -term mild jogging increases insulin action despite no influence on body mass index or VO2max. Journal of Applied Physiology, Washington DC, v.66, n.5, p.2206-2210, 1989.

PEREIRA, L.O., FRANCISCHI, R.P., KLOPFER, M., PERROTI, A.C., CAMPOS, P.L., SAWADA, L.A., COSTA, S.R., LANCHA JR., A.H. Different intensities of physical activities with or without hypocaloric diet: effects on body composition, food consumption and plasmatic profile in obese women. Medicine and Science in Sports and Exercise, Baltimore, v.30, n.5, p.238S, 1998. Supplement.

POPKIN, B.M., DOAK, C.M. The obesity epidemic is a worldwide phenomenon. Nutrition Reviews, Washington DC, v.56, n.4 (Pt 1), p.106-114, 1998.

RACETTE, S.B., SCHOELLER, D.A., KUSHNER, R.F., NEIL, K.M., HERLING-IAFFALDANO, K. Effects of aerobic exercise and dietary carbohydrate on energy expenditure and body composition during weight reduction in obese women. American Journal of Clinical Nutrition, Bethesda, v.61, n.3, p.486494, 1995a.

RACETTE, S.B., SCHOELLER, D.A., KUSHNER, R.F., NEIL, K.M. Exercise enhances dietary compliance during moderate energy restriction in obese women. American Journal of Clinical Nutrition, Bethesda, v.62, n.2, p.345-349, 1995b.

RAVUSSIN, E., BURNAND, B., SCHUTZ, Y., JÉQUIER, E. Energy expenditure before and during energy restriction in obese patients. American Journal of Clinical Nutrition, Bethesda, v.41, n.4, p.753-759, 1985.

ROLLS, B.J., SHIDE, D.J. The influence of dietary fat on food intake and body weight. Nutrition Reviews, Washington DC, v.50, n.10, p.283-290, 1992.

ROSS, R., PEDWELL, H., RISSANEN, J. Response of total and Regional lean tissue and skeletal muscle to a program of energy restriction and resistance exercise. International Journal of Obesity and Related Metabolic Disorders, Hampshire, v.19, n.4, p.781S-787S, 1995. Supplement.

RÖSSNER, S. Dietary fibre in the prevention and treatment of obesity. In: SCHWEIZER, T.F., EDWARDS, C.A. Dietary fibre: a component of food. London : Springer-Verlag, 1992. p.295-332.

SARIS, W.H.M. Exercise with or without dietary restriction and obesity treatment. International Journal of Obesity and Related Metabolic Disorders, Hampshire, v.19, n.4, p.113S116S, 1995. Supplement.

SHETTY, P.S. Physiological mechanisms in the adaptative response of metabolic rates to energy restriction. Nutrition Research Reviews, Cambridge, v.3, p.49-74, 1990.

SOCIEDADE BRASILEIRA DE CARDIOLOGIA. Exercício anti-sedentarismo/obesidade [online]. 1999. [citado em 14/4/ 99]. Disponível no endereço: <http://www.cardiol.br/ exerc.htm >.

SOCIEDADE BRASILEIRA DE CARDIOLOGIA. Programa 
nacional de prevenção e epidemiologia [online]. 1999. [citado em 14/4/99]. Disponível no endereço: <http:// www.cardiol.br/epidemio.htm $>$.

STOLL, B.A. Timing of weight gain in relation to breast cancer risk. Annals of Oncology, Dordrecht, v.6, n.3, p.245-248, 1995.

STUNKARD, A.J., WADDEN, T.A. Psychological aspects of human obesity. In: BJÖRNTORP, P., BRODOFF, B.N. Obesity. Philadelphia : J.B. Lippincott, 1992. p.352-360.

SWEENEY, M.E., HILL, J.O., HELLER, P.A., BANEY, R., DIGIROLAMO, M. Severe vs moderate energy restriction with and without exercise in the treatment of obesity: efficiency of weight loss. American Journal of Clinical Nutrition, Bethesda, v.57, n.2, p.127-134, 1993.

SWINBURN, B., RAVUSSIN, E. Energy balance or fat balance. American Journal of Clinical Nutrition, Bethesda, v.57, n.5, p.766S-771S, 1993. Supplement.

TRUSWELL, A.S., BEYNEN, A.C. Dietary fibre and plasma lipids: potential for prevention and treatment of hyperlidaemias. In: SCHWEIZER, T.F., EDWARDS, C.A. Dietary fibre: a component of food. London : Springer-Verlag, 1992. p.295332.

VAN ITALLIE, T.B., YANG, M. Cardiac dysfunction in obese dieters: a potentially lethal complication of rapid, massive weight loss. American Journal of Clinical Nutrition, Bethesda, v.39, n.5, p.695-702, 1984.

WALBERG, J.L. Aerobic exercise and resistance weight training during weight reduction. Implications for obese persons and athletes. Sports Medicine, Auckland, v.7, n.6, p.343-356, 1989.

WIRTH, A. Non-pharmacological therapy for metabolic syndrome. Herz, Munchen, v.20, n.1, p.56-69, 1995.

WORLD HEALTH ORGANIZATION. Diet, nutrition and the prevention of chronic diseases. Geneva, 1990. p.69-73. (Technical Report Series, 797).

Recebido para publicação em 28 de julho de 1998 e aceito em 28 de maio de 1999. 